\title{
Understanding Solution State Conformation and Aggregate Structure of Conjugated Polymers via Small Angle X-ray Scattering
}

\author{
Justin J. Kwok ${ }^{1 *}$, Kyung Sun Park ${ }^{2}$, Bijal B. Patel ${ }^{2}$, Rishat Dilmurat ${ }^{3}$, David Beljonne ${ }^{3}$, Xiaobing \\ $\mathrm{Zuo}^{4}$, Byeongdu Lee ${ }^{4}$ and Ying Diao ${ }^{1,2,5,6 *}$ \\ ${ }^{1}$ Department of Materials Science and Engineering, University of Illinois at Urbana-Champaign, \\ 1304 W. Green St., Urbana, IL 61801, USA. \\ ${ }^{2}$ Department of Chemical and Biomolecular Engineering, University of Illinois at Urbana- \\ Champaign, 600 S. Mathews Ave., Urbana, IL 61801, USA. \\ ${ }^{3}$ Laboratory for Chemistry of Novel Materials, University of Mons, Place du Parc, 20, B-7000 \\ Mons, Belgium. \\ ${ }^{4}$ Advanced Photon Source, Argonne National Laboratory, Lemont, Illinois 60439, USA. \\ ${ }^{5}$ Beckman Institute, Molecular Science and Engineering, University of Illinois at Urbana- \\ Champaign, 405 N. Mathews Ave., Urbana, IL 61801, USA. \\ ${ }^{6}$ Frederick Seitz Materials Research Laboratory, University of Illinois at Urbana-Champaign, \\ 104 S. Goodwin Ave., Urbana, IL 61801, USA
}

*Co-corresponding authors. Email: jikwok2@illinois.edu (J.J.K.), yingdiao@illinois.edu (Y.D.)

\begin{abstract}
Donor-acceptor (D-A) conjugated polymers are high-performance organic electronic materials that exhibit complex aggregation behavior. Understanding the solution state conformation and aggregation of conjugated polymers is crucial for controlling morphology during thin-film deposition and the subsequent electronic performance. However, a precise multiscale structure of solution state aggregates is lacking. Here, we present an in-depth small angle X-ray scattering (SAXS) analysis of the solution state structure of an isoindigo-bithiophene based D-A polymer (PII-2T) as our primary system. Modeling the system as a combination of hierarchical fibrillar aggregates mixed with dispersed polymers, we extract information about conformation and
\end{abstract}


multiscale aggregation and also clarify the physical origin of features often observed but unaddressed or misinterpreted in small-angle scattering patterns of conjugated polymers. The persistence length of the D-A polymer extracted from SAXS agrees well with a theoretical model based on the dihedral potentials. Additionally, we show that the broad high $q$ structure factor peak seen in scattering profiles can be attributed to lamellar stacking occurring within the fibril aggregates and that the low $q$ aggregate scattering is strongly influenced by the polymer molecular weight. Overall, the SAXS profiles of D-A polymers in general exhibit a sensitive dependence on the co-existence of fibrillar aggregate and dispersed polymer chain populations. We corroborate our findings from SAXS with electron microscopy of freeze-dried samples for direct imaging of fibrillar aggregates. Finally, we demonstrate the generality of our approach by fitting the scattering profiles of a variety of D-A polymers. The results presented here establish a picture of the D-A polymer solution state structure and provide a general method of interpreting and analyzing their scattering profiles.

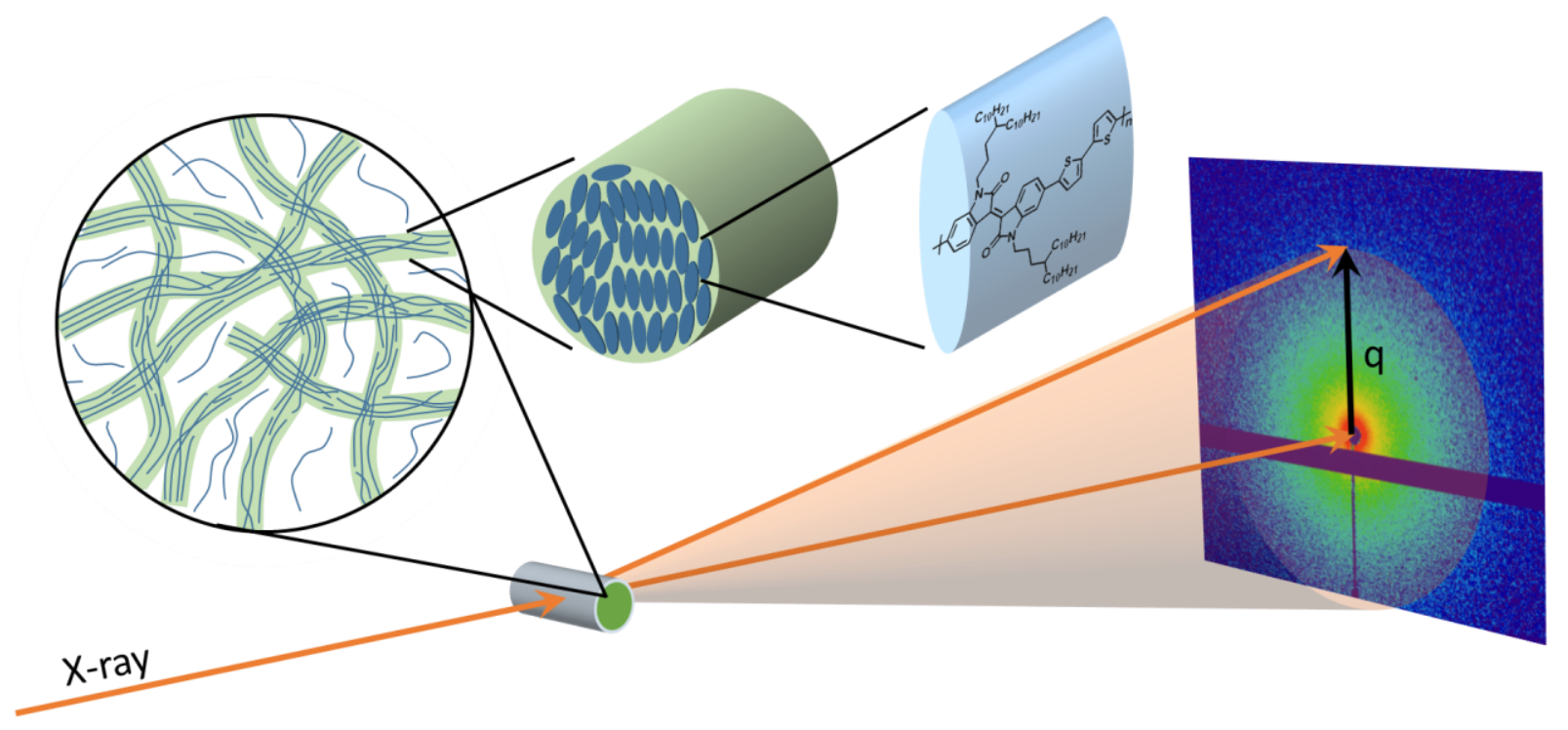




\section{Introduction}

One of the key advantages of conjugated polymers, and organic semiconductors in general, is their ability to be solution processed on a large scale with high throughput and energy efficiency. Since the performance and functionality of conjugated polymers is a direct consequence of their multiscale morphology it is incredibly important to understand and carefully control the structure of conjugated polymers at all length scales. Towards this end, preaggregation of conjugated polymer solutions prior to deposition has been studied extensively by means of solvent quality tuning and solution pre-processing, as it can drastically improve morphology and electronic performance ${ }^{1-8}$. However, many studies have been phenomenological, focusing on the tuning of processing parameters followed by ex situ characterization of film morphology or electronic device properties. Additionally, many such works fail to ascertain or investigate further the precise aggregate structure, instead relying on qualitative visual assessments of films or spectroscopic comparisons. While these studies have led to the development of high level design rules for optimizing performance, there is a lack of fundamental understanding regarding the actual solution state conformation and assembly structure of conjugated polymers. Furthermore, commonly used methods such as UV-Vis spectroscopy and ex situ film characterization give hints towards the polymer aggregation state in solution but they do not provide a clear picture. While small angle X-ray or neutron scattering (SAXS/SANS) can yield a detailed, quantitative description of the aggregation structure, the scattering profiles of D-A conjugated polymers have been difficult to interpret.

Although grazing incidence wide angle scattering (GIWAXS) of conjugated polymer thin films is a widely used technique for studying solid state crystallinity and orientation, SAXS/SANS has not been as widely used for characterizing conjugated polymer solutions. This 
may be due to technical challenges such as the high X-ray absorption cross-section of commonly used chlorinated solvents or the need for long exposure times and the subsequent issue of sample beam damage. Additionally, as conjugated polymers may exhibit complex assembly behavior, the analysis of scattering profiles is non-trivial and requires a careful interpretation. Despite this, there have been a few scattering studies that aim to uncover the conformation of conjugated polymers, most of which have focused on the second generation of conjugated homopolymers such as P3HT and MEH-PPV which are more flexible or can exist in a fully dissolved state ${ }^{9-18}$. The Pozzo group ${ }^{14,15}$ studied the hierarchical aggregation P3HT using SANS in different solvents and temperatures, characterizing the evolution of fully dissolved P3HT chains to parallelepipeds and fibril networks. McCulloch et al. ${ }^{17}$ was able to use the wormlike chain model to fit P3AT scattering, finding the persistence length to be $\sim 3 \mathrm{~nm}$ and decreasing with increasing temperature. While other works were not able to use physical models for the scattering, the presence of semiflexible chains and large aggregation was evident from power law scaling ${ }^{11,12}$. However, it remains difficult to interpret D-A conjugated polymer scattering due to strong aggregation and possible structure factor effects. Key limitations in previous studies include the sole use or misapplication of power law scaling methods or graphical methods such as Kratky plots as well as data with limited $q$-range that prevents developing a more complete picture of the solution state. Even among studies applying models to fit the scattering profile, there are inconsistencies between the reported values and their scattering data, likely due in part to challenges in accurate fitting and interpretation. Furthermore, the extracted physical parameters or inferred physical pictures were often not validated against alternative measurements.

Nonetheless, there are a couple of works that have successfully demonstrated SAXS/SANS modeling and an understanding of the D-A conjugated polymer solution. For 
example, the Pozzo group ${ }^{19}$ studied SANS of D-A polymers such as DPPDTT in a variety of solvents ranging from good to poor and polar to non-polar. Using a combined model consisting of parallelepipeds for nanoribbon aggregates and semiflexible cylinders for dispersed chains, they were able to show that while poor solvents induce aggregation, the polarity of the solvents was an important factor in enhancing nanoribbon aggregation as opposed to more amorphous aggregates in non-polar solvents. Liu and Boué et al. ${ }^{20}$ similarly used a parallelepiped and cylinder model to analyze SANS of DPPDTT finding the D-A polymer conformation to transition from rod-like chains in good solvents to ribbon-like aggregates and networks in poor solvents. While these works have successfully modeled and analyzed D-A polymer scattering, they have not observed the high $q$ structure factor peak that has been reported in several other studies ${ }^{21-23}$ and observed consistently in our work.

In this work, we present a holistic method for evaluating D-A polymer solution state primarily through SAXS experiments supported by scanning electron microscopy (SEM) and atomic force microscopy (AFM) imaging of freeze-dried solutions. To add to the current understanding of conjugated polymer solution state through scattering, we present here several modeling approaches towards understanding D-A polymer scattering and developing a clearer picture of the solution state. We present two models for the scattering profiles, the first of which is based on the contribution of two semiflexible cylinders similar to what has been previously reported but with a pseudo-Voigt peak to capture a lamellar peak feature (2SFC model). More importantly, we develop a second model based on the scattering theory of particle assemblies ${ }^{24,25}$ that explicitly considers hierarchical aggregation of polymer chains into fibril aggregates (FA model). In doing so, we are not only able to fit and extract structural parameters from scattering but also establish the physical basis for the unique features observed in D-A polymer scattering 
and thereby improve the understanding of conjugated polymer solution state aggregation. In particular, we resolve the origin of the broad high $q$ peak that has been intermittently reported in prior works ${ }^{21-23}$. While it has been speculated to be due to lamellar stacking it has not been clearly explained or modeled so far. In addition, it has not been observed consistently potentially due to limited $q$-range, high incoherent background scattering, or its dependence on solvent quality, as we will show. Furthermore, we demonstrate the generality of our fibril aggregation model by fitting a variety of D-A polymer scattering profiles containing the lamellar peak.

\section{Experimental}

\section{Materials and Molecular Weight}

The isoindigo-bithiophene-based polymer (PII-2T) and thieno-isoindigo-bithiophenebased polymer (PTII-2T) were synthesized as previously reported ${ }^{26,27}$. A low MW PII-2T $(\mathrm{Mn}=$ 19,883, $\mathrm{Mw}=61,700, \mathrm{PDI}=3.1)$ and $\mathrm{a}$ high $\mathrm{MW}$ PII-2T $(\mathrm{Mn}=30,645, \mathrm{Mw}=76,809, \mathrm{PDI}=$

2.5) batch were characterized using high temperature gel permeation chromatography at $150{ }^{\circ} \mathrm{C}$ in trichlorobenzene. The full molecular weight distributions of PII-2T are shown in Figure S1. PTII-2T molecular weight could not be measured due to strong interaction with the column material, even at high temperature. The polymer solutions were prepared by dissolving the polymer in either chlorobenzene (anhydrous, 99.8\%; Sigma-Aldrich Inc.), decane (anhydrous, $\geq 99 \%$; Sigma-Aldrich Inc.), or a mixture of both. The solutions were then stirred at $80{ }^{\circ} \mathrm{C}$ for at least 12 hours prior to characterization. After heating, the solutions were removed to room temperature for 15 minutes prior to being used in all experiments.

\section{Density Functional Theory (DFT) Calculations and Persistence Length}


In order to estimate the persistence length of our PII-2T polymer, we first calculate the dihedral potentials within the repeat unit using density functional theory (DFT) with global hybrid function B3LYP in conjunction with a 6-31G(d,p) basis set using Gaussian 16. The side chains were substituted with $\mathrm{CH}_{3}$ to reduce computational cost. Using the method demonstrated by Milner and Gomez et al. ${ }^{28}$, the dihedral potentials are then used to randomly generate a set of 500,000 polymer conformations (each with 20 repeat units) by rotating each rotatable segment in accordance with a Boltzmann distribution using the dihedral potential. A breakdown of the PII$2 \mathrm{~T}$ repeat unit along with the segment lengths and deflection angles are shown in Figure S2 and Table S1. The persistence length is then extracted from the decay of the average tangent-tangent correlation function

$$
\left\langle v_{0} \cdot v_{n}\right\rangle=e^{-n / n_{p}}
$$

Where $n$ is the number of the repeat unit, $v_{n}$ is the backbone tangent vector of repeat unit $n$ and $n_{p}$ is the persistence length in terms of repeat units. The persistence length in terms of length $l_{p}$ is determined by multiplying $n_{p}$ by the length of the PII-2T repeat unit which was determined from DFT simulations to be $\sim 1.8 \mathrm{~nm}$.

\section{Small Angle X-Ray Scattering (SAXS) experiments}

SAXS experiments were carried out at the 12-ID-B beamline of the Advanced Photon Source at Argonne National Laboratory using an X-ray beam energy of $13.3 \mathrm{keV}$. A Pilatus $2 \mathrm{M}$ detector was used at sample-to-detector distances of $2 \mathrm{~m}$ and $3.6 \mathrm{~m}$. The polymer solution SAXS experiments were performed using a flow cell to prevent beam damage and enable longer exposure times. The flow cell was constructed using a $1 \mathrm{~mm}$ diameter quartz capillary connected to PTFE tubing using PTFE heat shrink tubing. One tubing end was connected to a syringe pump 
which cycled the polymer solution back and forth through the capillary at a linear velocity of about $1 \mathrm{~mm} / \mathrm{s}$ within the capillary while a series of $0.1 \mathrm{~s}$ exposures with $3 \mathrm{~s}$ delays were accumulated for a total exposure time of approximately 1-2 s. Each polymer solution and its neat solvent background were scanned successively at the same position of the same flow cell in order to ensure that the instrumental "empty cell" scattering was consistent. Such procedure was necessary due to the weak scattering of our conjugated polymer relative to chlorinated solvent in order to ensure proper background subtraction. The isotropic 2D scattering patterns were then reduced, background subtracted, and averaged using the beamline's MATLAB package. The 1D scattering profiles were then analyzed and fit using custom models in SasView which are provided in the supplementary information.

\section{Freeze drying, Scanning Electron Microscopy (SEM) and Atomic Force Microscopy (AFM) Measurements}

In order to validate the SAXS results we use a freeze-drying method to directly image the structure of aggregates in solution, which are then imaged using AFM and SEM. For the freezedrying process $2 \mu \mathrm{L}$ of polymer solution were sandwiched between a bare Si substrate and a glass coverslip. The sandwiched sample is first submerged in liquid mixture of $63 \%$ propane, $37 \%$ ethane and then stored in liquid nitrogen. Since this mixture has a larger heat capacity than liquid nitrogen, it can quickly freeze the sample without boiling off and therefore prevent aggregation during the cooling process ${ }^{29}$. In samples frozen with liquid nitrogen alone, solvent crystallization is observed whereas samples frozen in the liquid propane/ethane mixture exhibit amorphous frozen solvent (as observed under cross-polarized optical microscopy). The top coverslip is separated while submerged in liquid nitrogen and the sample is then quickly transferred to a sealed Linkam stage chamber (LTS420) which is held at $-100{ }^{\circ} \mathrm{C}$ in a nitrogen 
atmosphere. The temperature is raised to $-80{ }^{\circ} \mathrm{C}\left(0.5^{\circ} \mathrm{C} / \mathrm{min}\right)$ for chlorobenzene $\left(\mathrm{mp}=-45^{\circ} \mathrm{C}\right)$ solutions and to $-60{ }^{\circ} \mathrm{C}$ for decane $\left(\mathrm{mp}=-30{ }^{\circ} \mathrm{C}\right)$ while vacuum is applied for $\sim 6$ hours in order to fully sublimate the solvent while preventing melting. The sample is imaged using tapping mode AFM (Asylum Research Cypher) and SEM (JEOL JSM-7000F) at $25 \mathrm{kV}$ accelerating voltage.

\section{Results and Discussion}

\section{Determination of Persistence Length and Chain Conformation}

First, we computationally determined the size and shape of singular PII-2T chains to establish a base level understanding of the potential chain conformation and to compare with experimental SAXS measurements. We first estimated the persistence length of the conjugated polymer using an inexpensive computational method demonstrated by Milner and Gomez et al. ${ }^{28}$

The dihedral potentials of the polymer repeat unit were calculated using DFT and a large number of conformations were then generated based on the lengths, deflection angles, and dihedral potentials determined by DFT. The average tangent-tangent correlation was then calculated to extract the persistence length. Three unique dihedral potentials were calculated within the PII-2T repeat unit as shown in Figure 1A corresponding to the intra-isoindigo, isoindigo-thiophene, and thiophene-thiophene bonds. The isoindigo-thiophene bond within the repeat unit is also equivalent to the bond connecting adjacent repeat units. The calculated dihedral potentials are shown in Figure 1B. PII-2T chain conformations were then randomly generated based on the dihedral potentials. An example of a 60-mer corresponding to the weight-averaged molecular weight of the polymer is shown in Figure 1C illustrating the semiflexible conformation of the 
polymer with each red or black segment representing a repeat unit. As shown in Figure 1D, a persistence length of $7.5 \mathrm{~nm}$ was extracted from the average tangent-tangent correlation of the random conformations. While there has not been any prior report of the persistence length of PII$2 \mathrm{~T}$, the estimated value here is in line with the persistence length of typical D-A polymers ${ }^{30}$ and also considering that PII-2T has relatively high torsion as shown by DFT in our previous work ${ }^{31}$. As discussed later, this value closely matches with the persistence length extracted from our SAXS experiments. Using the persistence length and molecular weight results, we then estimate the $R_{g}$ of a single PII-2T chain using the expression for a wormlike chain,

$$
R_{g}^{2}=\frac{l_{p} L}{3}-l_{p}^{2}+\frac{2 l_{p}^{3}}{L}\left(1-\frac{l_{p}}{L}\left(1-\exp \left(-\frac{L}{l_{p}}\right)\right)\right)
$$

finding $R_{g}$ to be $7 \mathrm{~nm}$ and $12 \mathrm{~nm}$ corresponding to the number-averaged and weight-averaged molecular weights, respectively. This result suggests that if PII-2T exists as dispersed polymer chains in dilute solution then the SAXS profile should exhibit a Guinier knee corresponding to the overall chain size at $q \sim 0.01 \AA^{-1}$ and a constant flat intensity below this value. 

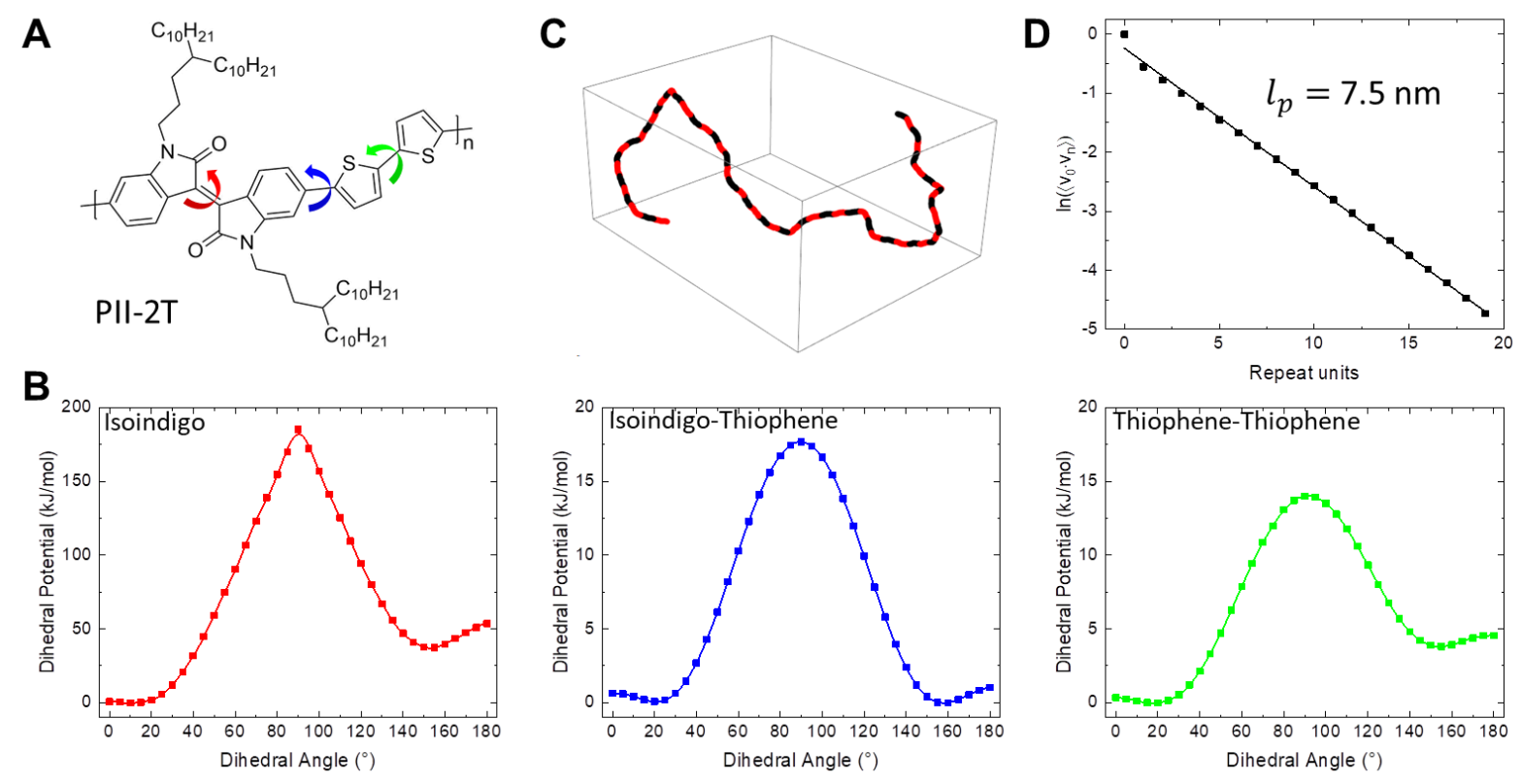

Figure 1. PII-2T dihedral potentials and persistence length calculation. A) PII-2T repeat unit with rotating bonds indicated for intra-isoindigo (red), isoindigo-thiophene (blue), and thiophene-thiophene (green). The bonds between repeat units are also isoindigo-thiophene bonds. B) Dihedral potentials calculated by DFT corresponding to the rotating bonds shown in the repeat unit. The points are the orientations sampled by DFT and the lines are empirical fits used for interpolation. C) Example of a randomly generated PII-2T conformation using the dihedral potentials. D) Average tangent-tangent correlation plot with extracted persistence length of 7.5 nm.

\section{Analysis of Low MW PII-2T Scattering Features}

SAXS experiments were carried out to determine the conformation and structure of PII$2 \mathrm{~T}$ in solution. A schematic of the SAXS experiment is shown in Figure 2A. As our primary system we use a low MW PII-2T dissolved in chlorobenzene (CB). CB was chosen as it is a fairly good solvent commonly used in solution processing of conjugated polymers. Other common solvents such as chloroform, dichlorobenzene, and trichlorobenzene have too large absorption cross-sections making it difficult to collect data with good signal-to-noise ratio. 
SAXS scattering profiles of low MW PII-2T in CB at 1, 2, 10, and $20 \mathrm{mg} / \mathrm{mL}$ are shown in Figure 2B. Several features are immediately apparent upon inspection of the SAXS profiles. First, at low $q\left(q<0.02 \AA^{-1}\right)$ a power law of $\sim-1.2$ to -1.6 is observed instead of an overall Guinier region preceded by constant flat intensity indicating that the PII-2T chains are not dispersed but are aggregating into a larger structure. Such low $q$ power law scattering and even steeper is commonly observed in small angle scattering of conjugated polymers ${ }^{11-14,21,22}$. Given the known tendency of PII-2T to form nanofibrils ${ }^{31}$ a slope of -1.2 to -1.6 at this length scale is expected, corresponding to a large semiflexible object in between that of a rigid rod $(-1)$ and random coil $(-2)$. We note that this low $q$ slope does vary slightly from experiment to experiment in the range of -1.2 to -1.6 indicating the exact aggregation structure is highly sensitive to the solution preparation conditions but still exhibits a semiflexible nature in all cases. Secondly, at high $q$, a broad peak feature at $q \sim 0.25 \AA^{-1}$ can be seen for all concentrations. This feature occurs at the same location as a sharp diffraction peak seen in transmission SAXS of solid PII-2T used as synthesized (Figure S3) indicating that it stems from a structure factor peak as opposed to a form factor Guinier knee. The location of this peak is also consistent with the first order lamellar peak seen in GIWAXS of PII-2T printed films as shown in our previous study $^{31}$. Thus this broad scattering feature corresponds to weakly ordered stacking in the alkyl side chain direction and we therefore refer to it as the lamellar peak. We note that while this peak is a precursor to the lamellar peak seen in films and occurs at similar $q$, it is sufficiently broad that the exact packing structure cannot be distinguished. This peak has been observed in several scattering studies but its origin was not explained ${ }^{21-23}$. The presence of the low $q$ slope and high $q$ structure factor peak both points towards the presence of aggregates in solution but more specifically the slope of -1.2 to -1.6 at low $q$ further points towards the formation of elongated 
aggregate structures such as nanofibrils. Following the low $q$ region is a Guinier knee at $q$ $0.025 \AA^{-1}$ which should therefore correspond to some cross-sectional dimension of the elongated aggregate which will be discussed below. Furthermore, these features are seen at all concentrations, as low as $1 \mathrm{mg} / \mathrm{mL}$ (which was the lowest concentration that could be measured with a strong enough signal to overcome the incoherent background scattering at high $q$ ). The overlap concentration $\mathrm{c}^{*}$ of low MW PII-2T is estimated to be $\sim 10-20 \mathrm{mg} / \mathrm{mL}$ based on the expression for wormlike chains ${ }^{32}$ and using the computed persistence length of $7.5 \mathrm{~nm}$, indicating that aggregation occurs even in dilute solution. For all concentrations, the SAXS profiles are quite similar indicating an identical structure and no concentration dependence, at least for the length scales probed here. For the remainder of this work, we use the $10 \mathrm{mg} / \mathrm{mL}$ profile as our primary system of low MW PII-2T in CB due to it having the best signal quality over the entire $q$-range.

In order to understand the structure at high $q$ without the presence of the lamellar peak we systematically varied the solvent from $\mathrm{CB}$ to decane (Dec) as shown in Figure 2C. All SAXS measurements for the $\mathrm{CB} / \mathrm{Dec}$ series were performed at concentrations of $2 \mathrm{mg} / \mathrm{mL}$ due to large agglomeration in Dec at higher concentrations. Solutions with higher Dec fraction exhibited higher viscosity and visible agglomeration by eye. For all solvent ratios, the Guinier knee at $q$ $0.025 \AA^{-1}$ remains present and is preceded by a power law with slope ranging from -1 to -1.8 , with generally steeper slopes in solutions with high Dec. Notably, as the Dec fraction is increased, the lamellar peak intensity reduces significantly. Surprisingly, as the lamellar peak disappears a second Guinier knee is revealed at $q \sim 0.1 \AA^{-1}$. We suspect that the disappearance of the lamellar peak with increasing Dec is due to the stronger interaction between Dec and the alkyl side chains as opposed to the conjugated core, therefore disrupting lamellar stacking held 
together by aggregated alkyl side chains. This hypothesis is consistent with the fact that the low $q$ intensity also reduces with increasing Dec since a reduced correlation between aggregating polymers reduces the scattering intensity of the overall aggregate feature at low $q$. This can be seen in Figure $2 \mathrm{C}$ where the reduced intensity of the low $q$ knee at $0.025 \AA^{-1}$ causes the effective, intermediate slope at $0.05 \AA^{-1}$ to become shallower. While it is possible that the side chains will have reduced contrast in Dec due to chemical similarity, we do not believe that such an effect would result in the disappearance of the lamellar peak as even if the side chains were contrast matched with Dec, correlations between the conjugated backbone would still exist. In this work, we use Dec solvent as a means to access the newly revealed Guinier knee. The effect of solvents on the D-A conjugated polymer side chain and backbone and their subsequent effect on aggregation and SAXS is subject to future study.

Looking at both Guinier knees at $\sim 0.025 \AA^{-1}$ and $\sim 0.1 \AA^{-1}$ it can be seen that they are preceded by slopes corresponding to elongated, semiflexible objects and are followed by steeper slopes of -3 or lower. Considering the relatively high rigidity of D-A conjugated polymers and their tendency to form fibril aggregates and considering the power law slopes before and after the Guinier knees it is reasonable to assume that these knees correspond to a cross-sectional feature of elongated objects. As a first analysis, we therefore extract the cross-sectional radii by linearization of the cross-sectional Guinier knees based on the equation below.

$$
I(q) \propto \frac{1}{q} \exp \left(-\frac{R_{g}^{2} q^{2}}{2}\right)
$$

Equation (3) can then be linearized by plotting $\ln [q I(q)]$ vs. $q^{2}$ in the region around the Guinier knee which yields a line of slope $-R_{g}^{2} / 2$ where in this particular case $R_{g}$ is related to the physical radius $R$ by $R_{g}^{2}=R^{2} / 2$. The cross-sectional Guinier plots for the knees at $\sim 0.025 \AA^{-1}$ 
and $\sim 0.1 \AA^{-1}$ are shown in Figure $2 \mathrm{D}$ and E, respectively. Based on the extracted radius values we determine that the Guinier knees at $\sim 0.025 \AA^{-1}$ and $\sim 0.1 \AA^{-1}$ correspond to the cross-sections of fibrillar aggregates and individual polymer chains, respectively. We find that the fibril radius $R_{f}$ is $6.4 \mathrm{~nm}$ in CB (diameter $\left.D_{f}=12.8 \mathrm{~nm}\right)$ and $5.4 \mathrm{~nm}$ in Dec $\left(D_{f}=10.8 \mathrm{~nm}\right)$ and that the polymer radius $R_{p}$ is $14 \AA$ in $\operatorname{Dec}\left(D_{p}=28 \AA\right)$. The polymer diameter of $28 \AA$ is in agreement with the lamellar stacking distance of $\sim 25 \AA$ in thin films ${ }^{31}$ with it being slightly larger likely due to increased disorder in solution and/or increased solvent swelling. The fibril diameters of $\sim 10$ $\mathrm{nm}$ are also consistent with imaging experiments as discussed below. This analysis indicates that while the conjugated polymers aggregate into fibrils, some population of the conjugated polymers remain dispersed or at least loosely interacting such that they are isolated enough to maintain their individual cross-section. We emphasize that these linearization based methods are not meant to provide precise measurements but are instead meant to illustrate the more evident structural features of our conjugated polymer system and provide a rationale for more complex modeling approaches discussed later in this paper where these cross-sectional parameters as well as other features are extracted. 

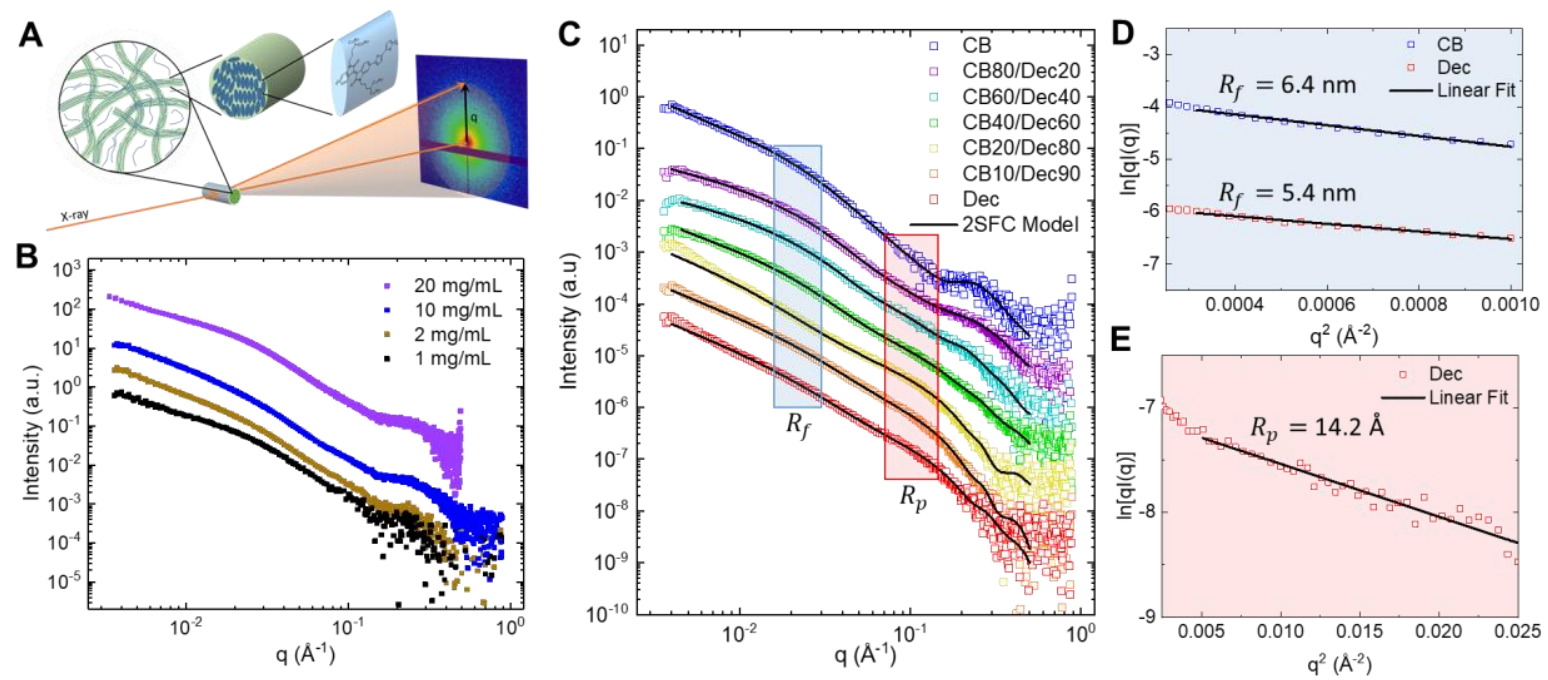

Figure 2. SAXS of low MW PII-2T in CB and Dec. A) Schematic of the SAXS experiment. B) SAXS profiles of low MW PII-2T in CB at 1, 2, 10, and $20 \mathrm{mg} / \mathrm{mL}$. C) SAXS profiles of low MW PII-2T in varying $\mathrm{CB} / \mathrm{Dec}$ solvent ratios. The low $q$ cross-sectional Guinier knee is highlighted in blue corresponding to the fibril radius $R_{f}$ and the high $q$ cross-sectional Guinier knee is highlighted in red corresponding to the polymer radius $R_{p}$. Cross-sectional Guinier plots for the fibril in blue (D) and polymer in red (E) with extracted radius parameters.

\section{Establishing Solution State Structure through Molecular Weight and Solvent Dependence}

To further build a picture of the conjugated polymer and its fibril aggregate structure we also performed SAXS of a high MW PII-2T in CB and Dec. For comparison, Figure 3A shows the SAXS profiles of the low MW PII-2T (top pair) and high MW PII-2T (bottom pair) in both CB and Dec. The low MW PII-2T scattering profiles correspond to the data shown previously in Figure $2 \mathrm{~B}$ and $\mathrm{C}$. Having established the picture so far as consisting of two semiflexible populations corresponding to fibrillar aggregates and polymer chains it may at first seem that the low and high MW solution states are quite different. However, by comparing the four conditions, we can in fact unify all scattering profiles under this interpretation and further expand our understanding of the solution state. First, the most obvious difference between the low and high 
MW solutions is that the low $q$ slope for high MW PII-2T solutions is much steeper at -3.3 . However, such a steep slope corresponds to interfacial Porod scattering and simply indicates the aggregate has become large enough that it's cross-sectional Guinier knee has moved below the accessible $q$-range as depicted by green curve and scheme in Figure 3A. On the other hand, the high $q$ region is very similar between the low and high MW scattering profiles, which can be seen in both CB solutions by the presence of the lamellar peak and even more clearly in both Dec solutions by the nearly identical high $q$ Guinier knee and slopes. In this case, the similarity indicates that the polymer cross-section remains the same regardless of molecular weight or aggregation, which is to be expected. Additionally, it indicates that the lamellar stacking is consistent as well. Overall, the dual aggregate and polymer population model is consistent with the molecular weight and solvent data. Based on these conclusions, we illustrate in Figure 3A below each MW pair a simplified scheme of green and blue curves corresponding to scattering of large and small elongated objects, respectively. The scheme illustrates the separate contributions of the large fibril aggregates and the smaller semiflexible polymer chains and how the shifting of the fibril contribution can produce the overall scattering curves seen by experiment for the low and high MW PII-2T. This interpretation is confirmed by our imaging results discussed below. For further discussion on how these scattering components can be treated additively, please see supplementary section S1.

We can further illustrate this interpretation by looking at how the scattering of fibril aggregates evolves as the aggregate size increases. This is shown in Figure S4 where the polymers are approximated as cylinders which then aggregate into fibrils of increasing size. The simulated scattering intensity is calculated from the square of the Fourier transform of the cylinders' excess scattering length density. As the fibril diameter increases, the cross-sectional 
Guinier knee shifts to lower $q$ until it is out of range and only contributes its trailing Porod scattering. At the same time a structure factor peak emerges at high $q$ due to the cross-sectional packing of the cylinders. This shows how the seemingly different scattering profiles of low and high MW PII-2T can both be explained by fibril aggregation. The small and large fibril scattering in Figure S4 (green and purple) resembles the scattering of low and high MW PII-2T in Figure 3A, respectively.

To support our picture of the conjugated polymer solution state, we use additional techniques such as SEM and AFM freeze-dried imaging and UV-Vis spectroscopy. By freezedrying the PII-2T solutions in a liquid propane-ethane mixture and sublimating the solvent we are able to preserve the solution state structure and image it using SEM (Figure 3B) and AFM (Figure 3C). The AFM measurements are used to measure the fibril height in the out of plane direction. AFM linecuts are indicated by the dashed cyan line with the corresponding height profile shown in Figure 3D. Linecuts were chosen in regions with few overlapping fibrils for more accurate measurements. For low MW PII-2T, SEM imaging reveals that in CB the conjugated polymer forms fibril structures in solution with fibril widths of $\sim 10$ to several 10 's of nm in plane. From AFM imaging, fibril heights out-of-plane are shown to be primarily from 10 to $20 \mathrm{~nm}$, which is in agreement with the in-plane width estimated from SEM and also the crosssectional Guinier plot in Figure 2D (yielding a diameter of $12.8 \mathrm{~nm}$ ). It is important to note that while the SEM imaging appears to show several fibril diameters larger than $\sim 10 \mathrm{~nm}$, the small number of these larger fibrils is not as pronounced in SAXS because SAXS is a numberaveraged method. The similar magnitude between fibril width and height for low MW PII-2T in $\mathrm{CB}$ indicates the fibrils have roughly isotropic cross-sections. For low MW PII-2T in Dec, SEM imaging shows two types of structures featuring large agglomerates consistent with observations 
by eye and also fibril aggregates with widths of $\sim 10 \mathrm{~nm}$ in plane. Likewise, AFM imaging shows fibril agglomeration and fibril aggregates with heights ranging from several $\mathrm{nm}$ to $\sim 20 \mathrm{~nm}$. For high MW PII-2T in CB, the SEM and AFM results show clearly increased fiber dimensions with the widths ranging from approximately several 10's of $\mathrm{nm}$ to $>100 \mathrm{~nm}$ and the heights ranging from $\sim 50$ to $100 \mathrm{~nm}$ in the AFM linecut. This is consistent with the fibril cross-sectional Guinier knee moving out of range below $q=0.003 \AA^{-1}$ that corresponds to fiber diameters of $>\sim 60 \mathrm{~nm}$. Furthermore, it supports the argument that the low $q$ scattering is due to Porod scattering of the aggregate's interface rather than from a dimensionality of 3. For high MW PII-2T in Dec the SEM image shows fibrils as well as a large agglomerated fibril network. The fibril width appears to range from several 10 's of $\mathrm{nm}$ to $100 \mathrm{~nm}$ in the agglomerate. AFM of the agglomerates shows heights of several 10's of $\mathrm{nm}$ as well. Additionally, what is quite interesting is that the large change in the degree of aggregation and agglomeration from low MW to high MW stems primarily from the increased polydispersity of the molecular weight distribution. Looking at the molecular weight distributions (Figure S1) the peak molecular weights are in fact the same and so the difference in the mean $\mathrm{Mn}$ and $\mathrm{Mw}$ values stem primarily from the existence or lack thereof of the low molecular weight tail which affects the polydispersity. We note that we observed features consistently $\sim 2.5 \mathrm{~nm}$ high but several 10's of nm wide in the AFM imaging of freeze-dried Dec solutions. This can be seen more clearly when scanning the samples at a higher magnification as shown in Figure S5. We attribute these features to adsorption during the sample preparation. For an extended discussion on these ribbon-like features and why they do not contribute to SAXS and likely do not exist in solution see supplementary section S2.

UV-Vis spectroscopy provides further validation for the observed sensitivity of aggregation on molecular weight. Normalized absorption spectra of the low and high MW PII-2T 
solutions in CB and Dec are shown in Figure S6. The major two peaks at $710 \mathrm{~nm}$ and $655 \mathrm{~nm}$ correspond to the $0-0$ and 0-1 transitions, respectively, while the peak at $420 \mathrm{~nm}$ corresponds to localized transitions from within the isoindigo moiety ${ }^{31}$. While the spectral shape can be predominantly captured by the vibronic progression of a single polymer chain ${ }^{33}$, the absorption peaks ratio $\mathrm{A}_{0-0} / \mathrm{A}_{0-1}$ is modified by $\mathrm{J}$ - and $\mathrm{H}$-aggregation character ${ }^{34}$. Comparing the low and high MW PII-2T solutions for either $\mathrm{CB}$ or Dec solvent, the $\mathrm{A}_{0-0} / \mathrm{A}_{0-1}$ ratio is larger for the high MW cases in both solvents indicating increased J-aggregation in agreement with enhanced fibril aggregation. Furthermore, comparing the $\mathrm{A}_{0-0} / \mathrm{A}_{0-1}$ ratio between $\mathrm{CB}$ and Dec solutions for both MWs shows Dec to have larger ratios indicating even further J-aggregation. Likewise, the red shifted 0-0 peak for high MW (compared to low MW) and for Dec solvent (compared to CB solvent) supports the presence of enhanced aggregation. 

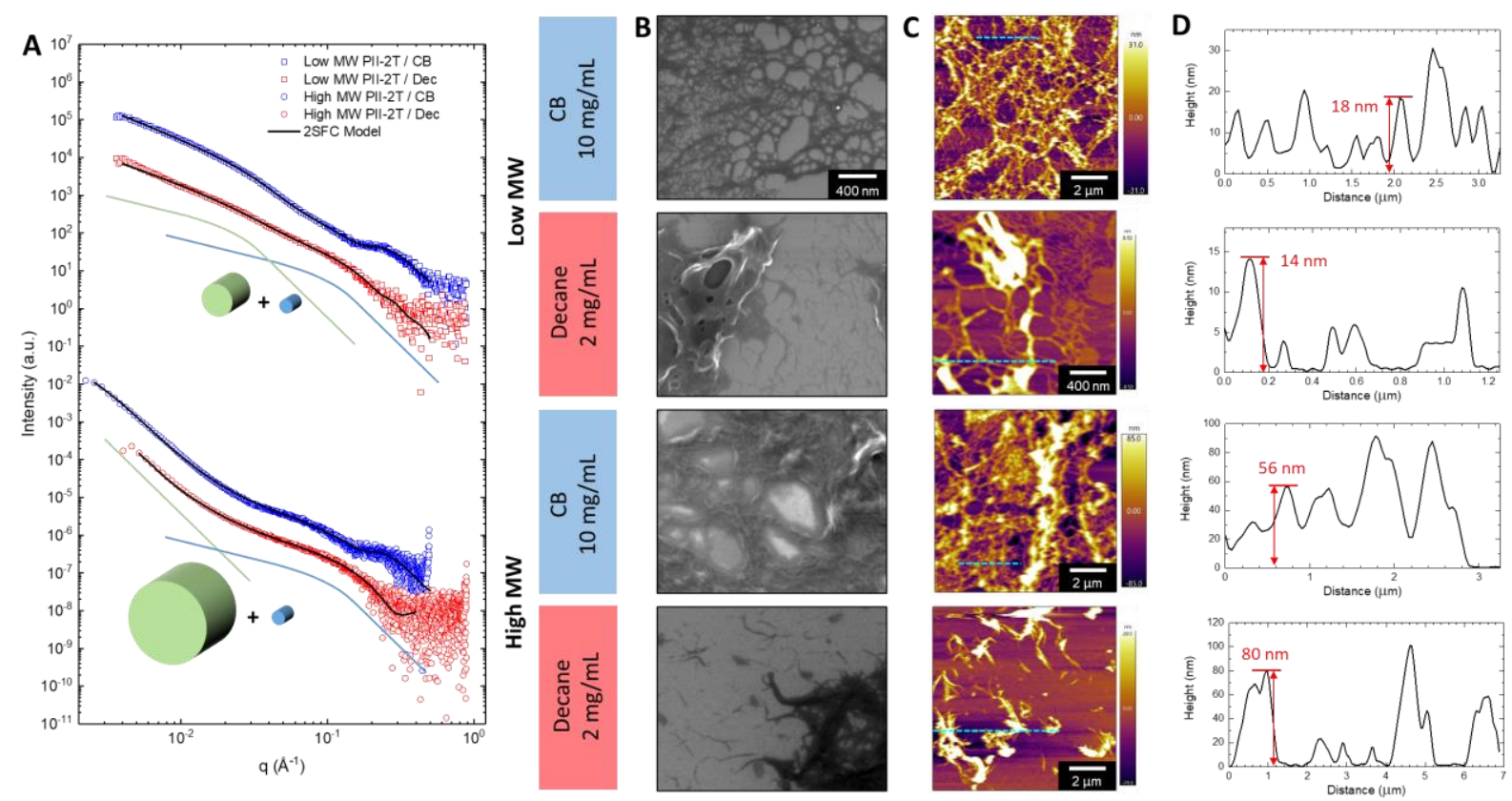

Figure 3. Molecular weight dependence of SAXS and solution state freeze-dried imaging and UV-Vis spectroscopy for PII-2T CB and Dec solutions. A) SAXS profiles of low and high MW PII-2T in CB and Dec along with a scheme demonstrating the underlying scattering features. 2SFC model fits are shown by the solid black line. Freeze-dried samples imaged by (B) SEM and (C) AFM long with (D) AFM linecuts demonstrating the height profile of the fibrillar aggregates.

So far, the careful analysis of SAXS profiles combined with supporting freeze-dried imaging and $\mathrm{UV}-\mathrm{Vis}$ spectroscopy results has allowed us to develop a clearer picture of the PII-2T solution state. In summary, the overall key points we have established so far are that:

- There exists two distinct populations in the observable SAXS range corresponding to fibrillar aggregates and dispersed polymers, both of which are elongated, semiflexible objects, 
- The broad structure factor peak seen in D-A polymer scattering stems from lamellar stacking of polymers within the aggregates indicating strong pre-aggregation at all conditions,

- The degree of aggregation and agglomeration is highly sensitive to the solvent but more interestingly to the molecular weight distribution of the polymer and its polydispersity.

\section{SAXS Model Development and Fitting}

Having established a picture of the conjugated polymer solution state we now develop full SAXS models to describe the SAXS profiles observed in this study and fully quantify the shape and structure of the fibrillar aggregates and dispersed polymer chains. Here, we present two SAXS models. The first is a more general model considering two independent populations of semiflexible cylinders with a pseudo-Voigt peak, which we refer to as the 2SFC (two semiflexible cylinder) model for short. The second is a more detailed model that explicitly considers that some population of the semiflexible polymer chains aggregate into fibrils, which we refer to as the FA (fibril aggregation) model for short. While the 2SFC model is more general, the FA model specifically accounts for the assembly of polymers and helps to understand the origin of broad structure factor peaks observed in conjugated polymer SAXS/SANS. A description of the models is discussed below with further details of the model theory found in supplementary section S3.

In the $2 \mathrm{SFC}$ model, we consider that two populations of scatterers are present. The first corresponds to large fibril aggregates while the second corresponds to polymer chains that could be either individually dispersed or consisting of a few chains loosely joined to produce a longer 
effective length but still maintaining its individual cross-section. Both of these populations are modeled as elongated semiflexible objects denoted by the subscripts $f$ and $p$ for fiber and polymer, respectively. Additionally, a pseudo-Voigt peak function is used to fit the lamellar structure factor peaks if present. The total model for the scattering intensity is then,

$$
\begin{gathered}
I(q)=A \Sigma_{C S, f}\left(q ; R_{f}\right) \Sigma_{S F C, f}\left(q ; b_{f}, L_{f}\right)+B \Sigma_{C S, p}\left(q ; R_{p}\right) \Sigma_{S F C, p}\left(q ; b_{p}, L_{p}\right) \\
+C V_{P}\left(q ; q_{c}, w, \mu\right)+D
\end{gathered}
$$

Where $\Sigma_{C S, i}$ is the shape function for the cross-sectional component of the semiflexible cylinder, $\Sigma_{S F C, i}$ is the shape function for the axial component of the semiflexible cylinder, $q$ is the scattering vector, $R_{i}$ is a function which describes cross-sectional radius, $b_{i}$ is the Kuhn length, which is twice the persistence length $l_{p, i}, L_{i}$ is the contour length, $V_{P}$ is the pseudo-Voigt peak function with peak center $q_{c}$, full width at half maximum (FWHM) $w$, and Lorentz fraction $\mu$. A, $B$, and $C$ are scaling factors that lump together various prefactors such as number density, contrast, volume, and experimental constants while $D$ is the background level if present. For the cross-sectional shape function $\Sigma_{C S, i}\left(q ; R_{i}\right)$, we use a circular cross-section. Additionally, we apply polydispersity to the fibril radius (and subsequent functions of it) using a Schulz-Zimm distribution. In this case, the specified fiber radius is the mean radius of the distribution (denoted by an overbar) and the parameter $\sigma_{f}$ describes the width of the fibril radius distribution. The relevant terms in equation (4) then represent averages according to the polydispersity distribution. While polydispersity may also be applied to the polymer radius, we find it unnecessary as the Guinier knee of the polymer radius either quickly approaches a background level or soon becomes dominated by the lamellar peak contribution. For the axial shape function 
of the semiflexible object $\Sigma_{S F C, i}\left(q ; b_{i}, L_{i}\right)$ we use the function developed by Pedersen and Schurtenberger ${ }^{35}$ for semiflexible polymers with excluded volume effects with corrections by Chen et al. ${ }^{36}$ as implemented by SasView ${ }^{37}$. Because the model primarily describes two separate populations of semiflexible cylinders corresponding to the fiber and polymer we refer to it as the two semiflexible cylinder (2SFC) model in this work. In some cases, if the radius of the fibril aggregates is so large that its Guinier knee is below the experimental $q$-range then only the trailing Porod region will be observed at low $q$. In this case the polydisperse fibril radius can be set to a sufficiently large value out of range, which effectively makes its contribution equivalent to $q^{-4}$. Alternatively, if aggregation or network formation is expected but the exact form of the aggregation is unknown then the fibril term in equation (4) can be replaced with a power law function $A q^{-d}$ where the exponent $d$ will describe the shape or dimensionality of the aggregate at that $q$-range. In this work we find $d$ to be between 3.77 to 4 in the case of large aggregates thus indicating it is indeed specifically due to interfacial Porod scattering.

The 2SFC model is general enough to fit a variety of conjugated polymer scattering profiles, primarily D-A polymers that exhibit fibril aggregation due to high rigidity. In order to fit potential structure factor peaks that appear, a pseudo-Voigt function is used in order to extract the peak position and FWHM that can give information such as d-spacing and coherence length. For some cases this is sufficient, however there are two shortcomings with this model. The first is that it only considers the external shape of the fibrils and ignores that they are composed of polymer chains and the second is that it does not explain the origin of the lamellar structure factor peak that has been seen in D-A conjugated polymer scattering ${ }^{21-23}$. To address these issues we developed the FA model based on small angle scattering theory for particle assemblies ${ }^{24,25}$. As the fibrils are composed of polymer chains assembled together, we use this theory to describe 
the fibril cross-section as consisting of an assembly of primary polymer chain cross-sections. Here we take the fibril cross-section to be circular while the assembled polymers exhibit an elliptical cross-section due to the anisotropic packing of conjugated polymers where lamellar stacking occurs in the direction of the side chains. Considering a population of fibrils composed of aggregated polymers as well as a population of dispersed polymers, the total scattering intensity is then

$$
\begin{gathered}
I(q)=A \Sigma_{C S, p}^{\mathrm{ell}}\left(q ; R_{p}, \varepsilon\right)\left[\gamma N_{p} \Sigma_{C S, f}\left(q ; R_{f}\right)+S_{H S}\left(q ; 2 R_{p}, \phi_{H S}\right)\right] \Sigma_{S F C, f}\left(q ; b_{f}, L_{f}\right) \\
+B \Sigma_{C S, p}\left(q ; R_{p}\right) \Sigma_{S F C, p}\left(q ; b_{p}, L_{p}\right)+D
\end{gathered}
$$

In this case, $R_{p}$ serves as both the radius of dispersed polymer and also the elliptical major radius of the assembled polymers (minor radius $\varepsilon R_{p}$ ) and $\gamma$ is the contrast ratio $\Delta \rho_{f}^{2} / \Delta \rho_{p}^{2}$. The radius of the fibril cross-section $R_{f}$ is then calculated from $R_{p}$ by equating areas using $R_{f}=\left(\varepsilon R_{p}^{2} N_{p} /\right.$ $\left.\phi_{p}\right)^{1 / 2}$ where $N_{p} / \phi_{p}$ is treated as a single parameter and corresponds to the number of polymers within a fibril cross-section divided by the packing fraction. Because $\phi_{p}$ is the cross-sectional packing fraction of polymers within the fibril aggregate it is approximately 1 and therefore $N_{p} \sim N_{p} / \phi_{p}$, which gives an estimate of the number of polymers that constitute the aggregate's cross-section. For the internal structure factor we use a Schulz-Zimm distributed Percus-Yevick hard sphere structure factor ${ }^{38} S_{H S}$ with an effective spacing of $D_{e f f}=2 R_{p}$ and an effective volume fraction $\phi_{H S}$. This therefore couples the lamellar stacking distance to the major diameter of the polymer. Polydispersity is then applied to $R_{p}$ and all subsequent functions of $R_{p}$ using a Schulz-Zimm distribution with width parameter $\sigma_{p}$ and mean radius $\overline{R_{p}}$. The relevant terms in equation (5) are then averages according to the distribution (denoted by an overbar). We find 
that the hard sphere structure factor is sufficient to model the lamellar peak due to the fact that the fibril term $\gamma N_{p} \Sigma_{C S, f}$ dominates the internal structure factor (the term in brackets) at low $q$ and the large polydispersity also drastically smooths out the features of the internal structure factor which occur at high $q$. We find that the overall structure factor $S(q)=\gamma N_{p} \Sigma_{C S, f}+S_{H S}$ developed with this theory is similar to the structure factor for explicitly positioned, monodisperse, rigid cylinder bundles ${ }^{39}$ (Figure S7) but is of course more generally applicable. While $\pi-\pi$ stacking likely occurs if lamellar stacking is present we do not consider it as it is not observable in dilute solution SAXS as it is too weak to overcome the incoherent background in the WAXS region.

The scattering of the low and high MW PII-2T in CB solution is fitted to the 2SFC model shown in Figure 4A and B, respectively. The individual contributions are shown as well, shifted down slightly for visibility. We find that for low MW PII-2T in CB the mean fibril diameter $\overline{R_{f}}$ and polymer diameter $\overline{R_{p}}$ is $10.3 \mathrm{~nm}$ and $31.2 \AA$, respectively, which is in good agreement with the initial cross-sectional Guinier analysis and freeze-dried imaging results. Likewise, for high MW PII-2T in CB we find a mean fibril diameter of $78 \mathrm{~nm}$ and a polymer diameter of $30.6 \AA$, also in good agreement (the extracted fibril diameter can be treated as a lower bound). From the Voigt peak center $q_{c}$ we can also determine the lamellar d-spacing to be $\sim 28 \AA$ for both low and high MW PII-2T in CB which is in agreement with the polymer diameters and the lamellar stacking distance in solid PII-2T. We also found that the Lorentzian fraction of the pseudo-Voigt peak $\mu$ nearly always converged to 1 . Although peaks are convolutions of Gaussian and Lorentzian character, systems with large paracrystalline disorder will approach a purely Lorentzian shape ${ }^{40}$, which is reasonable for pre-aggregates formed in solution. Fit parameters for the 2SFC model for low and high MW PII-2T in CB and Dec are shown in Table 1. In addition, 
since more of the polymer scattering is revealed in the high MW data we are able to fit the persistence length $l_{p, p}$ resulting in values of 7.9 and $6.5 \mathrm{~nm}$ in CB and Dec, respectively, close to our calculated value of $7.5 \mathrm{~nm}$. For the low MW PII-2T we fix the persistence length to $7.5 \mathrm{~nm}$ since the model is rather insensitive to its value due to the overshadowing presence of fibril aggregate scattering at intermediate $q$. Alternatively, we also show that for the high MW PII-2T the larger SFC contribution can be replaced with a power law if one wishes to apply a more general approach. Fitting with the power law is summarized in Figure S8 and Table S2, with the low $q$ power law slope being -3.77 and -3.9 for $\mathrm{CB}$ and Dec, respectively, which is close to the ideal Porod slope of -4 and confirms our previous conclusion that the scattering at low $q$ stems from interacial scattering of a separate population of large aggregates rather than due to a structural dimension. Additionally, we use the $2 \mathrm{SFC}$ model to fit the $\mathrm{CB} / \mathrm{Dec}$ series shown in Figure 2C. A summary of the fit parameters for the $\mathrm{CB} / \mathrm{Dec}$ series can be found in the supplementary (Table S3).

In order to address the origin of the lamellar peak scattering and the fact that fibril aggregates are composed of polymers, we now use the FA model which explicitly considers such hierarchical composition based on scattering theory of particle assemblies ${ }^{24,25}$. A scheme is shown in Figure 4C demonstrating the model. It is assumed that polymers exhibit lamellar stacking within the fibril aggregate which also gives rise the structure factor peak at high $q$. We note that the scheme is simplified and that the polymer aggregate may not possess such high degree of positional or orientation order as is drawn. Fitting of the FA model to low and high MW PII-2T in CB is shown in Figure 4C and D along with the individual contributions. The fibril scattering contributes to both the low $q$ scattering and the high $q$ peak as a result of the new structure factor with respect to the primary polymer, $S(q)=\gamma N_{p} \Sigma_{C S, f}+S_{H S}$. Because the fibril 
contribution encompasses both the low $q$ aggregate scattering and high $q$ peak this clearly shows that the high $q$ peak is indeed a structure factor peak that stems from lamellar stacking within the fibril aggregate. Fit parameters of the FA model are shown in Table 2 along with the mean fibril radius $\overline{R_{f}}$ derived from the parameters showing similar results for the fibril and polymer radii as compared to the $2 \mathrm{SFC}$ model. These quantitative results also validate that the high $q$ peak originates from internal lamellar stacking within the fibril aggregates. Since the fibril radius $\overline{R_{f}}=\left(\varepsilon R_{p}^{2} N_{p} / \phi_{p}\right)^{1 / 2}$ is dependent on the product of $\varepsilon$ and $N_{p} / \phi_{p}$, one can be specified to determine the other under an additional constraint. Fits with the FA model can be made by fixing $\varepsilon$ to 0.13 (the ratio of $\pi-\pi$ to lamellar stacking distance) forcing a polymer minor diameter of $\sim 4$ $\AA$, corresponding to the $\pi-\pi$ stacking distance. This gives an estimate for the number of polymers within the fibril cross-section, $N_{p} \sim N_{p} / \phi_{p}$. FA model fits with $\varepsilon$ fixed to 0.13 are shown in Figure S9 with results in Table S4. A scheme is shown in Figure 4E depicting the key dimensions of the PII-2T solution state structure which consists of both fibrils composed of polymer chains and a separate polymer population. We again emphasize that the persistence length extracted from SAXS matches with our theoretical calculation and that the extracted dimensions agree with our imaging results as well. 

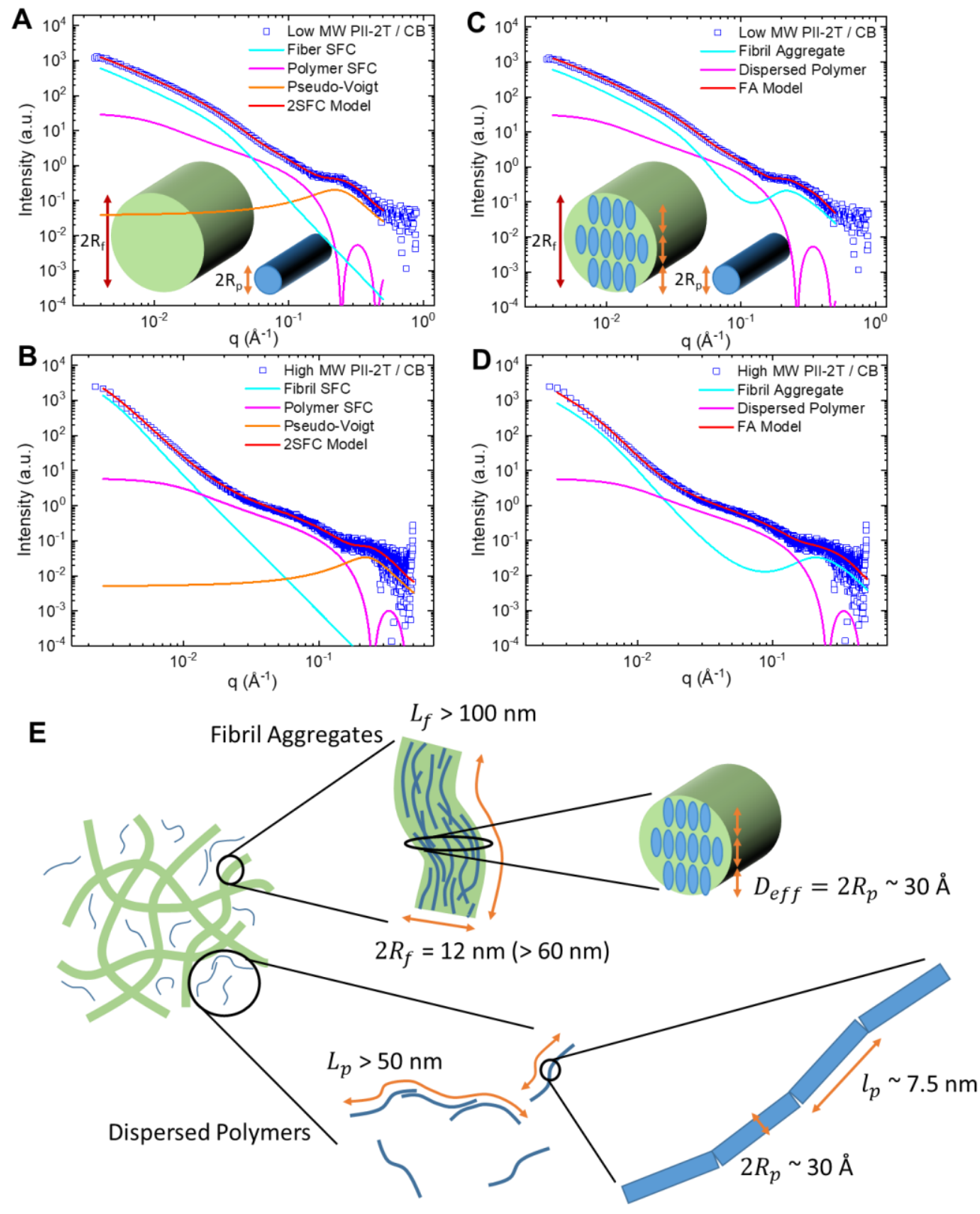

Figure 4. Model fitting of PII-2T in CB with solution state scheme. Low and high MW PII-2T in CB SAXS profiles fitted with (A,B) the 2SFC model and (C,D) the FA model. Individual contributions are shifted down for visibility. (E) Scheme of fibril aggregation and polymer populations for PII-2T in CB with parameters extracted from fits. Values in parenthesis are for high MW PII-2T only.

Table 1. Fitting parameters for 2SFC model. 


\begin{tabular}{|c|c|c|c|c|c|c|c|c|c|}
\hline Sample & $\begin{array}{c}L_{f} \\
(\mathrm{~nm})\end{array}$ & $\begin{array}{c}l_{p, f} \\
(\mathrm{~nm})\end{array}$ & $\begin{array}{c}\overline{\overline{R_{f}}} \\
(\mathrm{~nm})\end{array}$ & $\sigma_{f}$ & $\begin{array}{c}L_{p} \\
(\mathrm{~nm})\end{array}$ & $\begin{array}{c}l_{p, p} \\
(\mathrm{~nm})\end{array}$ & $R_{p}(\AA)$ & $q_{c}\left(\AA^{-1}\right)$ & $w\left(\AA^{-1}\right)$ \\
\hline Low MW PII-2T / CB & $500^{*}$ & $12 \pm 1$ & $5.15 \pm 0.08$ & $0.41 \pm 0.03$ & $100 *$ & $7.5^{*}$ & $15.6 \pm 0.7$ & $\begin{array}{c}0.220 \pm \\
0.007\end{array}$ & $\begin{array}{c}0.21 \pm \\
0.01\end{array}$ \\
\hline High MW PII-2T / CB & $500 *$ & $>R_{f}$ & $39 \pm 2$ & $0.70 \pm 0.02$ & $100 *$ & $7.9 \pm 0.9$ & $15.3 \pm 0.3$ & $\begin{array}{c}0.221 \pm \\
0.004\end{array}$ & $\begin{array}{c}0.185 \pm \\
0.008\end{array}$ \\
\hline Low MW PII-2T / Dec & $500 *$ & $13.2 \pm 0.4$ & $4.1 \pm 0.7$ & $0.60 \pm 0.2$ & $100 *$ & $7.5^{*}$ & $12.1 \pm 0.6$ & - & - \\
\hline High MW PII-2T / Dec & $500^{*}$ & $>R_{f}$ & $36 \pm 5$ & $0.47 \pm 0.05$ & $100 *$ & $6.5 \pm 0.4$ & $11.8 \pm 0.2$ & - & - \\
\hline
\end{tabular}

*Value was fixed during fitting.

Table 2. Fitting parameters for FA model.

\begin{tabular}{|c|c|c|c|c|c|c|c|c|c|c|c|}
\hline Sample & $\begin{array}{c}L_{f} \\
(\mathrm{~nm})\end{array}$ & $\begin{array}{c}l_{p, f} \\
(\mathrm{~nm})\end{array}$ & $\varepsilon$ & $N_{p} / \phi_{p}$ & $\begin{array}{c}\overline{R_{f}} \\
(\mathrm{~nm})\end{array}$ & $\begin{array}{c}L_{p} \\
(\mathrm{~nm})\end{array}$ & $\begin{array}{l}l_{p, p} \\
(\mathrm{~nm})\end{array}$ & $\begin{array}{l}\overline{R_{p}} \\
(\AA)\end{array}$ & $\sigma_{p}$ & $\phi_{H S}$ & $\gamma N_{p}$ \\
\hline $\begin{array}{c}\text { Low MW PII-2T / } \\
\text { CB }\end{array}$ & $500^{*}$ & $8.4 \pm 0.5$ & $0.25 \pm 0.02$ & $44 \pm 6$ & $4.8 \pm 0.4$ & $100^{*}$ & $7.5^{*}$ & $14.7 \pm 0.3$ & $0.36 \pm 0.01$ & $0.65 \pm 0.02$ & $3.9 \pm 0.5$ \\
\hline $\begin{array}{c}\text { High MW PII-2T / } \\
\text { CB }\end{array}$ & $500 *$ & $>R_{f}$ & $0.25 \pm 0.05$ & $2000 \pm 500$ & $34 \pm 5$ & $100^{*}$ & $9 \pm 1$ & $15.0 \pm 0.4$ & $0.44 \pm 0.02$ & $0.69 \pm 0.03$ & $60 \pm 20$ \\
\hline
\end{tabular}

*Value was fixed during fitting.

\section{Generality of SAXS Models and Analysis}

To demonstrate the generality of our scattering models, we applied the model fitting and analysis to a variety of D-A conjugated polymer SAXS. We collected and modeled SAXS profiles of the conjugated polymers PTII-2T, DPP2T-TT, and DPP-BTz that we used in our previous study ${ }^{31}$. Information on these material systems can be found in the Experimental section and in the Figure 5 caption. In addition, we visually extracted and modeled scattering profiles of other conjugated polymers published in previous works, including PDPP2FT-C 16 , P3HT- $b$ DPPT-T, and P(NDI2OD-T2) ${ }^{21-23}$. FA model fits and their individual contributions are shown for the conjugated polymers in Figure 5 with parameters summarized in Table S5. Information on the material systems of the extracted data can be found in the figure caption. PTII-2T in CB shows similar scattering to high MW PII-2T in CB (large aggregates) although the Guinier knee corresponding to the dispersed population occurs as lower $q$. As a result the mean radius of the polymer is larger at $22.1 \AA$. Additionally the intermediate slope corresponding to the 
semiflexibility of dispersed chains is nearly -1 indicating rod-like rigidity which is in agreement with the high degree of planarity of PTII- $2 \mathrm{~T}^{31}$. As a result, the persistence length is significantly larger than the radius $l_{p, p} \gg R_{p}$ but its exact value cannot be reliably extracted in this asymptotic limit or with the available $q$-range. For DPP2T-TT in CB, while the scattering profile is similar to PTII-2T and high MW PII-2T, the apparent slope at $\sim 0.015 \AA^{-1}$ is in fact shallower than -1 meaning that the intermediate feature cannot be due to the persistence length of the polymer as the shallowest possible slope possible would be -1 if the polymer were a rigid rod. Therefore, contrary to other polymers the intermediate Guinier knee at $0.03 \AA^{-1}$ must correspond to the overall size of the polymer as opposed to its cross-section as seen by the low $q$ power law slope of 0 in the dispersed polymer contribution. This allows us to extract the contour length of the polymer. The extracted parameters show that the polymer should have a contour length $L_{p}$ of $\sim 15.5 \mathrm{~nm}$, a mean radius $\overline{R_{p}}$ of $18.5 \AA$, and a persistence length $l_{p, p}$ of $9.3 \mathrm{~nm}$ which is in agreement with previously reported theoretical values of persistence length ${ }^{30}$. For DPP-BTz in $\mathrm{CB}$, the scattering pattern was fit to the FA model without any dispersed polymer contribution indicating that nearly all of the polymers are in an aggregated state. The fibril aggregate has a diameter $D_{f}$ of $6.2 \mathrm{~nm}$ and exhibits rod-like behavior as the fibril persistence length must be significantly larger than the radius to exhibit an effective slope of $-1\left(l_{p, f} \gg R_{f}\right)$. For PDPP2FT$\mathrm{C}_{16}$ in $\mathrm{CB}$ and P3HT- $b$-DPPT-T in o-dichlorobenzene (DCB), a similar solution state structure to high MW PII-2T, PTII-2T, and DPP2T-TT is seen consisting of contributions from large fibril aggregates and dispersed polymers. The extracted persistence lengths are $20 \mathrm{~nm}$ and $4.7 \mathrm{~nm}$ for PDPP2FT-C $\mathrm{C}_{16}$ and P3HT- $b$-DPPT-T, respectively. The relatively high persistence length of PDPP2FT- $\mathrm{C}_{16}$ is consistent with the fact that the DPP unit is flanked by furans resulting in reduced backbone torsion and higher planarity in comparison to a structure with flanking 
thiophenes ${ }^{41-44}$. The low persistence length of P3HT- $b$-DPPT-T is likely a result of the incorporation of the relatively flexible P3HT block into the backbone which has a persistence length of $\sim 3 \mathrm{~nm}^{17,30}$. An alternative fit for P3HT- $b$-DPPT-T is also possible where the intermediate Guinier knee stems from the aggregate instead of the dispersed polymer (Figure S10, Table S6). For P(NDI2OD-T2) in DCB nearly all the polymers are in an rod-like fibril aggregate state similar to DPP-BTz. In all cases, we were able to fully model and analyze the scattering of the D-A conjugated polymers, including the lamellar structure factor peak at high $q$.
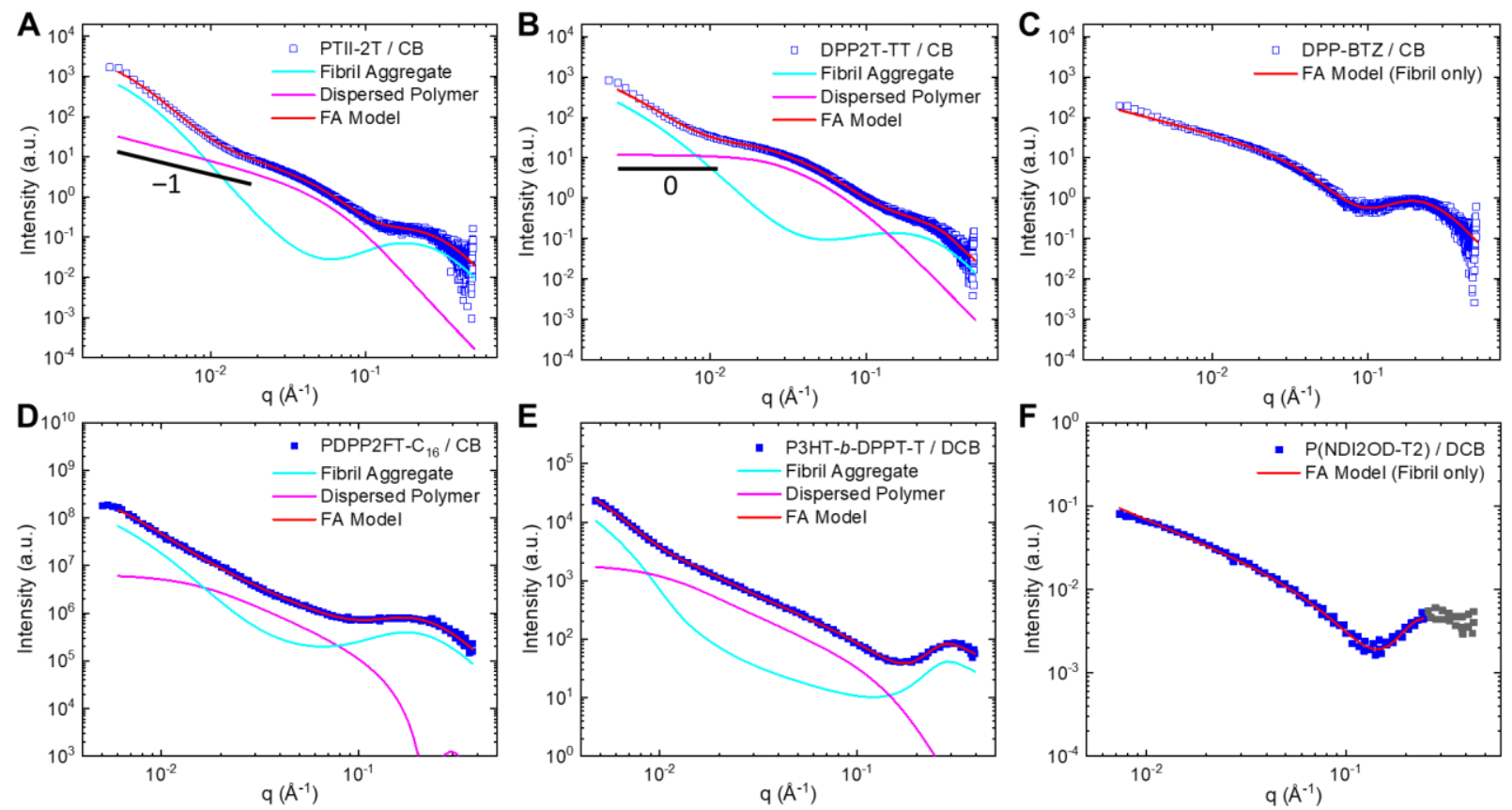

Figure 5. FA model fits for other D-A conjugated polymers in $\mathrm{CB}$ with individual contributions: (A) PTII-2T in CB at $20 \mathrm{mg} / \mathrm{mL}$ (similar to $10 \mathrm{mg} / \mathrm{mL}$ ), (B) DPP2T-TT in CB at $60 \mathrm{mg} / \mathrm{mL}$ (similar to 10 and $100 \mathrm{mg} / \mathrm{mL}$ ), (C) DPP-BTz in CB at $60 \mathrm{mg} / \mathrm{mL}$ (similar to 10 and $100 \mathrm{mg} / \mathrm{mL}$ ), (D) PDPP2FT-C 16 in $\mathrm{CB}$ at $1 \mathrm{wt} \%$, (E) P3HT- $b$-DPPT-T in DCB at $15 \mathrm{mg} / \mathrm{mL}$, and (F) $\mathrm{P}(\mathrm{NDI} 2 \mathrm{OD}-\mathrm{T} 2)$ in DCB at $5 \mathrm{mg} / \mathrm{mL}$. Individual contributions are shifted down for visibility. For $\mathrm{P}(\mathrm{NDI} 2 \mathrm{OD}-\mathrm{T} 2)$ the high $q$ region in grey was unreliably extracted due to overlapping data in the original source. 


\section{Conclusion}

In summary, we report the solution state of PII-2T and other D-A conjugated polymers consists of fibril aggregates in addition to a population of dispersed polymers. We showed through using various SAXS scattering profiles at different MW and solvents that the solution state conformation and aggregate structure can be inferred through careful analysis. We presented two models to explain the small angle scattering of D-A conjugated polymers which exhibit complex pre-aggregated behavior. The 2SFC model was generally applicable and was well suited to fitting the scattering profiles, yielding parameters in good agreement with freezedried imaging results. Likewise, the FA model also yielded parameters in good agreement with imaging results. Using the FA model we were able to demonstrate that the origin of the structure factor peak was due to side chain stacking from polymers within the fibril aggregates. To support our picture of the solution state and the applicability of our models, we carried out direct imaging of freeze-dried solution to visually compare the structure and size of fibril aggregates with our interpretation, finding good agreement. Finally, we demonstrated the generality of our model by successfully applying our model to a variety of different D-A conjugated polymers with scattering features distinct from those of PII-2T thus having differing solution state conformation and aggregation. This study has shown that pre-aggregation behavior of D-A conjugated polymers is complex but can be elucidated through the use of small angle scattering along with supporting UV-Vis spectroscopy and direct freeze-dried imaging. We present models as generally applicable tools to help interpret and analyze complex D-A polymer scattering, with 2SFC model as a more general but empirical model and the FA model as a new model based on SAXS theory of aggregation. Ultimately, the results presented here will assist in the 
understanding of conjugated polymer solution state aggregation and conformation which is a crucial aspect in controlling the structure and properties of conjugated polymers during solution processing.

\section{Acknowledgements}

J.J.K and Y.D. acknowledge support by the NSF CAREER award under grant \#18-47828. J.J.K. also acknowledges support from the U.S. Department of Energy, Office of Science, Office of Workforce Development for Teachers and Scientists, Office of Science Graduate Student Research (SCGSR) program. The SCGSR program is administered by the Oak Ridge Institute for Science and Education for the DOE under contract number DE-SC0014664. The work in Mons was supported by the European Union's Horizon 2020 research and innovation programme under Marie Sklodowska Curie Grant agreement No.722651 (SEPOMO project). Computational resources were provided by the Consortium des Équipements de Calcul Intensif (CÉCI), funded by the Fonds de la Recherche Scientifiques de Belgique (F.R.S.-FNRS) under Grant No. 2.5020.11, as well as the Tier-1 supercomputer of the Fedération Wallonie-Bruxelles, infrastructure funded by the Walloon Region under Grant Agreement No. 1117545. D.B. is a FNRS Research Director. This research used resources of the Advanced Photon Source, a U.S. Department of Energy (DOE) Office of Science User Facility, operated for the DOE Office of Science by Argonne National Laboratory under Contract No. DE-AC02-06CH11357. Extraordinary facility operations were supported in part by the DOE Office of Science through the National Virtual Biotechnology Laboratory, a consortium of DOE national laboratories focused on the response to COVID-19, with funding provided by the Coronavirus CARES Act. 


\section{References}

(1) Luzio, A.; Criante, L.; D’Innocenzo, V.; Caironi, M. Control of Charge Transport in a Semiconducting Copolymer by Solvent-Induced Long-Range Order. Scientific Reports 2013, 3 (1), 3425. https://doi.org/10.1038/srep03425.

(2) Wang, G.; Persson, N.; Chu, P.-H.; Kleinhenz, N.; Fu, B.; Chang, M.; Deb, N.; Mao, Y.; Wang, H.; Grover, M. A.; Reichmanis, E. Microfluidic Crystal Engineering of $\pi$-Conjugated Polymers. ACS Nano 2015, 9 (8), 8220-8230. https://doi.org/10.1021/acsnano.5b02582.

(3) Choi, D.; Chang, M.; Reichmanis, E. Controlled Assembly of Poly(3-Hexylthiophene): Managing the Disorder to Order Transition on the Nano- through Meso-Scales. Advanced Functional Materials 2015, 25 (6), 920-927. https://doi.org/10.1002/adfm.201403708.

(4) Chu, P.-H.; Kleinhenz, N.; Persson, N.; McBride, M.; Hernandez, J. L.; Fu, B.; Zhang, G.; Reichmanis, E. Toward Precision Control of Nanofiber Orientation in Conjugated Polymer Thin Films: Impact on Charge Transport. Chem. Mater. 2016, 28 (24), 9099-9109. https://doi.org/10.1021/acs.chemmater.6b04202.

(5) Li, M.; An, C.; Marszalek, T.; Baumgarten, M.; Yan, H.; Müllen, K.; Pisula, W. Controlling the Surface Organization of Conjugated Donor-Acceptor Polymers by Their Aggregation in Solution. Advanced Materials 2016, 28 (42), 9430-9438. https://doi.org/10.1002/adma.201602660.

(6) Gross, Y. M.; Trefz, D.; Tkachov, R.; Untilova, V.; Brinkmann, M.; Schulz, G. L.; Ludwigs, S. Tuning Aggregation by Regioregularity for High-Performance n-Type P(NDI2OD-T2) DonorAcceptor Copolymers. Macromolecules 2017, 50 (14), 5353-5366. https://doi.org/10.1021/acs.macromol.7b01386.

(7) Li, M.; Bin, H.; Jiao, X.; Wienk, M. M.; Yan, H.; Janssen, R. A. J. Controlling the Microstructure of Conjugated Polymers in High-Mobility Monolayer Transistors via the Dissolution Temperature. Angewandte Chemie International Edition 2020, 59 (2), 846-852. https://doi.org/10.1002/anie.201911311.

(8) Wu, Y.; Schneider, S.; Walter, C.; Chowdhury, A. H.; Bahrami, B.; Wu, H.-C.; Qiao, Q.; Toney, M. F.; Bao, Z. Fine-Tuning Semiconducting Polymer Self-Aggregation and Crystallinity Enables Optimal Morphology and High-Performance Printed All-Polymer Solar Cells. J. Am. Chem. Soc. 2020, 142 (1), 392-406. https://doi.org/10.1021/jacs.9b10935.

(9) Rawiso, M.; Aime, J. P.; Fave, J. L.; Schott, M.; Müller, M. A.; Schmidt, M.; Baumgartl, H.; Wegner, G. Solutions of Polydiacetylenes in Good and Poor Solvents : A Light and Neutron Scattering Study. J. Phys. France 1988, 49 (5), 861-880. https://doi.org/10.1051/jphys:01988004905086100.

(10) Aime, J. P.; Bargain, F.; Schott, M.; Eckhardt, H.; Miller, G. G.; Elsenbaumer, R. L. Structural Study of Doped and Undoped Polythiophene in Solution by Small-Angle Neutron Scattering. Physical Review Letters 1989, 62 (1), 55-58. https://doi.org/10.1103/PhysRevLett.62.55.

(11) Ou-Yang, W.-C.; Chang, C.-S.; Chen, H.-L.; Tsao, C.-S.; Peng, K.-Y.; Chen, S.-A.; Han, C. C. Micellelike Aggregates in Solutions of Semirigid Hairy-Rod Polymers. Phys. Rev. E 2005, 72 (3), 031802. https://doi.org/10.1103/PhysRevE.72.031802.

(12) Li, Y.-C.; Chen, K.-B.; Chen, H.-L.; Hsu, C.-S.; Tsao, C.-S.; Chen, J.-H.; Chen, S.-A. Fractal Aggregates of Conjugated Polymer in Solution State. Langmuir 2006, 22 (26), 11009-11015. https://doi.org/10.1021/la0612769.

(13) Kar Choudhury, P.; Bagchi, D.; Suchand Sangeeth, C. S.; Menon, R. Modified Conformation and Physical Properties in Conducting Polymers Due to Varying Conjugation and Solvent Interactions. Journal of Materials Chemistry 2011, 21 (5), 1607-1614. https://doi.org/10.1039/C0JM02304C.

(14) Newbloom, G. M.; Kim, F. S.; Jenekhe, S. A.; Pozzo, D. C. Mesoscale Morphology and Charge Transport in Colloidal Networks of Poly(3-Hexylthiophene). Macromolecules 2011, 44 (10), 38013809. https://doi.org/10.1021/ma2000515. 
(15) Newbloom, G. M.; Weigandt, K. M.; Pozzo, D. C. Structure and Property Development of Poly(3Hexylthiophene) Organogels Probed with Combined Rheology, Conductivity and Small Angle Neutron Scattering. Soft Matter 2012, 8 (34), 8854-8864. https://doi.org/10.1039/C2SM26114F.

(16) Keum, J. K.; Xiao, K.; Ivanov, I. N.; Hong, K.; Browning, J. F.; Smith, G. S.; Shao, M.; Littrell, K. C.; Rondinone, A. J.; Andrew Payzant, E.; Chen, J.; Hensley, D. K. Solvent Quality-Induced Nucleation and Growth of Parallelepiped Nanorods in Dilute Poly(3-Hexylthiophene) (P3HT) Solution and the Impact on the Crystalline Morphology of Solution-Cast Thin Film. CrystEngComm 2013, 15 (6), 1114-1124. https://doi.org/10.1039/C2CE26666K.

(17) McCulloch, B.; Ho, V.; Hoarfrost, M.; Stanley, C.; Do, C.; Heller, W. T.; Segalman, R. A. Polymer Chain Shape of Poly(3-Alkylthiophenes) in Solution Using Small-Angle Neutron Scattering. Macromolecules 2013, 46 (5), 1899-1907. https://doi.org/10.1021/ma302463d.

(18) Morgan, B.; Dadmun, M. D. The Importance of Solvent Quality on the Modification of Conjugated Polymer Conformation and Thermodynamics with Illumination. Soft Matter 2017, 13 (15), 27732780. https://doi.org/10.1039/C6SM02631A.

(19) Xi, Y.; Wolf, C. M.; Pozzo, L. D. Self-Assembly of Donor-Acceptor Conjugated Polymers Induced by Miscible 'Poor' Solvents. Soft Matter 2019, 15 (8), 1799-1812. https://doi.org/10.1039/C8SM02517G.

(20) Liu, C.; Hu, W.; Jiang, H.; Liu, G.; Han, C. C.; Sirringhaus, H.; Boué, F.; Wang, D. Chain Conformation and Aggregation Structure Formation of a High Charge Mobility DPP-Based DonorAcceptor Conjugated Polymer. Macromolecules 2020, 53 (19), 8255-8266. https://doi.org/10.1021/acs.macromol.0c01646.

(21) Schmidt, K.; Tassone, C. J.; Niskala, J. R.; Yiu, A. T.; Lee, O. P.; Weiss, T. M.; Wang, C.; Fréchet, J. M. J.; Beaujuge, P. M.; Toney, M. F. A Mechanistic Understanding of Processing AdditiveInduced Efficiency Enhancement in Bulk Heterojunction Organic Solar Cells. Advanced Materials 2014, 26 (2), 300-305. https://doi.org/10.1002/adma.201303622.

(22) Brady, M. A.; Ku, S.-Y.; Perez, L. A.; Cochran, J. E.; Schmidt, K.; Weiss, T. M.; Toney, M. F.; Ade, H.; Hexemer, A.; Wang, C.; Hawker, C. J.; Kramer, E. J.; Chabinyc, M. L. Role of Solution Structure in Self-Assembly of Conjugated Block Copolymer Thin Films. Macromolecules 2016, 49 (21), 8187-8197. https://doi.org/10.1021/acs.macromol.6b01686.

(23) Nahid, M. M.; Welford, A.; Gann, E.; Thomsen, L.; Sharma, K. P.; McNeill, C. R. Nature and Extent of Solution Aggregation Determines the Performance of P(NDI2OD-T2) Thin-Film Transistors. Advanced Electronic Materials 2018. https://doi.org/10.1002/aelm.201700559.

(24) Senesi, A. J.; Lee, B. Small-Angle Scattering of Particle Assemblies. Journal of Applied Crystallography 2015, 48 (4), 1172-1182. https://doi.org/10.1107/S1600576715011474.

(25) Li, X.; Charaya, H.; Bernard, G. M.; Elliott, J. A. W.; Michaelis, V. K.; Lee, B.; Chung, H.-J. LowTemperature Ionic Conductivity Enhanced by Disrupted Ice Formation in Polyampholyte Hydrogels. Macromolecules 2018, 51 (7), 2723-2731. https://doi.org/10.1021/acs.macromol.7b02498.

(26) Lei, T.; Dou, J.-H.; Pei, J. Influence of Alkyl Chain Branching Positions on the Hole Mobilities of Polymer Thin-Film Transistors. Advanced Materials 2012, 24 (48), 6457-6461. https://doi.org/10.1002/adma.201202689.

(27) Pruissen, G. W. P. V.; Gholamrezaie, F.; Wienk, M. M.; Janssen, R. A. J. Synthesis and Properties of Small Band Gap Thienoisoindigo Based Conjugated Polymers. J. Mater. Chem. 2012, 22 (38), 20387-20393. https://doi.org/10.1039/C2JM34668K.

(28) Zhang, W.; Gomez, E. D.; Milner, S. T. Predicting Chain Dimensions of Semiflexible Polymers from Dihedral Potentials. Macromolecules 2014, 47 (18), 6453-6461. https://doi.org/10.1021/ma500923r.

(29) Tivol, W. F.; Briegel, A.; Jensen, G. J. An Improved Cryogen for Plunge Freezing. Microsc Microanal 2008, 14 (5), 375-379. https://doi.org/10.1017/S1431927608080781.

(30) Kuei, B.; Gomez, E. D. Chain Conformations and Phase Behavior of Conjugated Polymers. Soft Matter 2017, 13 (1), 49-67. https://doi.org/10.1039/C6SM00979D. 
(31) Park, K. S.; Kwok, J. J.; Dilmurat, R.; Qu, G.; Kafle, P.; Luo, X.; Jung, S.-H.; Olivier, Y.; Lee, J.K.; Mei, J.; Beljonne, D.; Diao, Y. Tuning Conformation, Assembly, and Charge Transport Properties of Conjugated Polymers by Printing Flow. Science Advances 2019, 5 (8), eaaw7757. https://doi.org/10.1126/sciadv.aaw7757.

(32) Ying, Qicong.; Chu, Benjamin. Overlap Concentration of Macromolecules in Solution. Macromolecules 1987, 20 (2), 362-366. https://doi.org/10.1021/ma00168a023.

(33) Balooch Qarai, M.; Chang, X.; Spano, F. C. Vibronic Exciton Model for Low Bandgap DonorAcceptor Polymers. J. Chem. Phys. 2020, 153 (24), 244901. https://doi.org/10.1063/5.0029193.

(34) Chang, X.; Balooch Qarai, M.; Spano, F. C. HJ-Aggregates of Donor-Acceptor-Donor Oligomers and Polymers. J. Chem. Phys. 2021, 155 (3), 034905. https://doi.org/10.1063/5.0054877.

(35) Pedersen, J. S.; Schurtenberger, P. Scattering Functions of Semiflexible Polymers with and without Excluded Volume Effects. Macromolecules 1996, 29 (23), 7602-7612. https://doi.org/10.1021/ma9607630.

(36) Chen, W.-R.; Butler, P. D.; Magid, L. J. Incorporating Intermicellar Interactions in the Fitting of SANS Data from Cationic Wormlike Micelles. Langmuir 2006, 22 (15), 6539-6548. https://doi.org/10.1021/la0530440.

(37) Doucet, M.; Cho, J. H.; Alina, G.; Attala, Z.; Bakker, J.; Bouwman, W.; Butler, P.; Campbell, K.; Cooper-Benun, T.; Durniak, C.; Forster, L.; Gonzales, M.; Heenan, R.; Jackson, A.; King, S.; Kienzle, P.; Krzywon, J.; Nielsen, T.; O’Driscoll, L.; Potrzebowski, W.; Prescott, S.; Ferraz Leal, R.; Rozycko, P.; Snow, T.; Washington, A. SasView Version 5.0.2. https://doi.org/10.5281/zenodo.3752443.

(38) Botet, R.; Kwok, S.; Cabane, B. Percus-Yevick Structure Factors Made Simple. J Appl Cryst 2020, 53 (6), 1570-1582. https://doi.org/10.1107/S1600576720014041.

(39) Oster, G.; Riley, D. P. Scattering from Cylindrically Symmetric Systems. Acta Crystallographica 1952, 5 (2), 272-276. https://doi.org/10.1107/S0365110X5200071X.

(40) Rivnay, J.; Mannsfeld, S. C. B.; Miller, C. E.; Salleo, A.; Toney, M. F. Quantitative Determination of Organic Semiconductor Microstructure from the Molecular to Device Scale. Chemical Reviews 2012, 112 (10), 5488-5519. https://doi.org/10.1021/cr3001109.

(41) Yiu, A. T.; Beaujuge, P. M.; Lee, O. P.; Woo, C. H.; Toney, M. F.; Fréchet, J. M. J. Side-Chain Tunability of Furan-Containing Low-Band-Gap Polymers Provides Control of Structural Order in Efficient Solar Cells. J. Am. Chem. Soc. 2012, 134 (4), 2180-2185. https://doi.org/10.1021/ja2089662.

(42) Luzio, A.; Fazzi, D.; Nübling, F.; Matsidik, R.; Straub, A.; Komber, H.; Giussani, E.; Watkins, S. E.; Barbatti, M.; Thiel, W.; Gann, E.; Thomsen, L.; McNeill, C. R.; Caironi, M.; Sommer, M. Structure-Function Relationships of High-Electron Mobility Naphthalene Diimide Copolymers Prepared Via Direct Arylation. Chem. Mater. 2014, 26 (21), 6233-6240. https://doi.org/10.1021/cm503033j.

(43) Matsidik, R.; Martin, J.; Schmidt, S.; Obermayer, J.; Lombeck, F.; Nübling, F.; Komber, H.; Fazzi, D.; Sommer, M. C-H Arylation of Unsubstituted Furan and Thiophene with Acceptor Bromides: Access to Donor-Acceptor-Donor-Type Building Blocks for Organic Electronics. J. Org. Chem. 2015, 80 (2), 980-987. https://doi.org/10.1021/jo502432e.

(44) Matsidik, R.; Luzio, A.; Askin, Ö.; Fazzi, D.; Sepe, A.; Steiner, U.; Komber, H.; Caironi, M.; Sommer, M. Highly Planarized Naphthalene Diimide-Bifuran Copolymers with Unexpected Charge Transport Performance. Chem. Mater. 2017, 29 (13), 5473-5483. https://doi.org/10.1021/acs.chemmater.6b05313. 